experience based upon observation in the human being is very meagre. It is curious that such a lesion existed in such clinical conditions, there being no other focus of disease in the brain. That such an isolated lesion, occupying the grey matter, should be due to atheroma is highly improbable.

Carmarthen.

\section{A CASE OF \\ CHRONIC INVERSION OF THE UTERUS OF SEVEN MONTHS' DURATION SUCCESS- FULLY TREATED BY AVELING'S REPOSITOR.}

BY THOMAS OLIVER, M.D. GLASG., F.R.C.P. LOND., PHYSICIAN TO THE ROYAL INFIRMARY, NEWOASTLE-ON-TYNE.

ACUTE inversion of the uterus, if not restored, is said by some authors to be usually fatal. Under all circumstances it is a serious accident. My experience of inversion of the uterus has been gained in consultation with medical friends shortly after parturition in patients, in whom when an anæsthetic has been administered and the exposed surface of the womb has been rendered thoroughly antiseptic, I have had little difficulty in reducing the displacement. On the other hand, I have reported the case of a womb inverted for 18 years becoming malignant; so that, while inversion of the uterus is at all times a serious mishap, there is apparently a certain percentage of women who, once they get over the immediate effects of acute inversion, can go through life with the organ turned inside out without experiencing more than inconvenience.

Of the three different stages into which inversion of the uterus is divided $I$ need not give a lengthened description. They are briefly (1) where the fundus ateri does not come through the os externum; (2) where part of the inverted organ escapes beyond the os ; and (3) where the whole womb along with the cervix is inverted.

"Chronic inversion" is the term applied to an acute inversion that has not been reduced or to a displacement which has been slowly brought about by conditions apart from parturition Inversion, however, is mostly a sequence of parturition and is due to an enlarged and relaxed uterus with well-dilated cervix, upon the fundus of which pressure has been exerted during violent straining, or there has been a precipitate labour and a short umbilical cord, or the accoucheur has pulled too firmly upon the cord while the placenta is still adherent to the uterine wall. Its occurrence after a confinement is usually accompanied by a great amount of shock to the patient and by considerable hæmorrhage. The diagnosis is made by there being no longer felt any fundus above the pubes and in the worst forms by observing the inverted organ, in the form of a globular tumour of the size of a cocoanut, lying between the woman's thighs. The proper treatment at once suggests itself-viz., to replace the uterus under an anæsthetic, having previously taken all antiseptic precautions.

Opinion is divided as to whether if the after-birth is still adherent to the uterus it should be peeled off before attempting to reduce the inversion or whether the uterus should be reduced first and then the placenta removed. It is certainly easier to reduce the inverted organ without the attached placenta, but in peeling off the placenta before replacing the uterus there is often very profuse bleeding. Once the womb is restored the vagina is packed with iodoform gauze. Even where the uterus has been completely inverted after parturition for two or three days and its internal surface has become grey and unhealthy, the discharge escaping from it foul, and the temperature high, yet under thorough antiseptic precautions I have had excellent results notwitbstanding the extremely anæmic condition of the patient from loss of blood.

Most of the cases of acute inversion of the uterus are successfully treated at the time; s'me of the patients, on the other hand, die, while in a few the womb remains inverted. For the present I need only mention that inversion may be also due to a myoma that has passed through a dilated cervix and dragged the interior of the womb down too. Just as hæmorrhage is a symptom of acute inversion, so, also, is it common in chronic inversion. In this form the sym ptoms are recurrent hæmorrhage along with pain, a sense of dragging, and great discomfort.

A married woman, aged 24 years, was sent to me on
Nov. 14th, 1900, by Mr. E. O. Drake of Spennymoor, complaining of pain in the lower part of the abdomen, of frequent losses of blood, and of what she believed to be the womb protruding through the valva. She stated that she had been confined of her second child on May 1st, 1900, and that a midwife had attended her. The labour was easy and the after-birth came away readily without any traction having been made upon the cord. A few hours after delivery she passed a large clot which was followed by the rather sudden descent of the womb itself, accompanied by a very free escape of blood. Three days after this accident $\mathbf{M r}$. Drake was called in. Finding the uterus inverted and the patient very ill he tried to replace the organ, but only with partial success; nor was a second attempt the day after followed by any better result. The patient refused to allow any further efforts at restoration to be made and by degrees she recovered from the collapse and from the continuous bleeding. Her recovery, if it ever could be called such, was incomplete, for she began to have recurrent hæmorrhage which at times was rather profuse, and she became unable to attend to her domestic duties. At her menstrual periods the inverted uterus protruded further out of the vulva than in the intervals. She was in great pain and was so inconvenienced by the inverted nterus that she begged to be relieved. The patient was a slim woman and extremely pale and haggard-looking. The heart, the lungs, and the kidneys were healthy. On examination per vaginam the canal was found to be occupied by a pyriform tumour which was felt projecting through a widely dilated os uteri. The narrowed part of the tumour could be traced up inside the os and was found to be continuous with all that remained of the lower segment of the uterus. The sound passed up by the side of the tumour through the os for tbree-quarters of an inch. As the patient was of rather a spare build the hand could be driven well down into the abdominal cavity behind the pubes but no fundus conld be felt. Absence of the fundus above the pubes and the extremely short distance the sound penetrated through the widely dilated os made the diagnosis of chronic inversion of the uterus certain as against that of polypus. The case was not a prolapsed nterus, as was demonstrated to the students in the wards.

At my first examination of the patient I tried to reduce the displaced organ then and there, but it was too painful for the patient. On Nov. 22nd an anæsthetic was administered, when, after having carefully carbolised my hands, and having douched the vagina, I made an attempt both by central and lateral taxis to replace the organ, but although part of the womb could be replaced within the os I did not succeed in returning the whole organ. Failing thus to keep the fundus in its place, I applied Aveling's repositor, packed the vagina with iodoform ganze, and drew together the suspenders of the instrument. The repositor was left in situ for three days, Dr. W. Simpson, the senior house physician at the Newcastle Royal Infirmary, tightening the bands daily. When the repositor was removed the inversion had entirely disappeared, the steady and continuous pressure of the repositor having accomplished what forcible taxis failed to do. When I examined the patient on Nov. 28th and the following days no portion of the inverted fundus could be felt in the vagina. The finger could be carried through the os into a well-developed cervical canal nearly one inch in length and the uterine sound now entered to half an inch beyond the bend. The restoration of the uterus was quickly followed by signs of improvement in the general health of the patient. After a further few days rest in bed she left the infirmary feeling remarkably well with her uterus in the normal position.

Newcastle-on-Tyne.

\section{THE RELATION OF THE BOWEL LESION OF TYPHOID FEVER TO THE GENERAL SYMPTOMS OF THE DISEASE.}

By T. J. MACLAGAN, M.D. EDIN.

THE most prominent of the general symptoms of typhoid fever, that which from first to last is the dominant feature of the illness, is fever, indicated by a rise in the temperature of the body. This rise begins with the onset of the stage of invasion, reaches its maximum often as early as the fourth 
or fifth day, and generally by the end of the first week; after the tenth day there is as a rule no further rise, but the final fall of commencing convalescence seldom shows itself before the twentieth or twenty-first day of the fever. The temperature is higher in the evening than in the morning, the maximum range varying from $102^{\circ}$ to $104^{\circ} \mathrm{F}$. That is the course of the fever in an ordinary typical case of typhoid fever, in which the lesion goes on to sloughing and ulceration, in which no complications arise, and in which we have to deal with nothing except the action of the typhoid bacillus.

In very mild cases in which the inflammation of the intestinal glands terminates in resolution the temperature seldom rises beyond $101^{\circ}$ or $102^{\circ}$, and the fever comes to an end in from 12 to 20 days. In severe cases in which the fever lasts for four or five weeks the temperature may remain high during the whole course of the illness. Between the very severe and very mild cases there are all shades and degrees. There is no ailment which presents such protean features as typhoid fever, and no form of fever in which the temperature varies more in different cases. But in uncomplicated cases of typhoid fever the maximum range of temperature is reached before the tenth day, before the local lesion has given evidence of its existence.

How does the bacillus produce the general febrile disturbance? Is the fever merely symptomatic of the bowel lesion? Or is there any other agency at work? How the bacillus acts in producing the bowel lesion we have already seen. ${ }^{1}$ We have seen, too, that in its early stages, while affecting only the submucous coat, this lesion gives rise to no local disturbance, no local indications, subjective or objective, by which its existence could be diagnosed, and yet it is at this stage that the temperature attains its maximum. A local lesion which does not cause enough local disturbance to enable us to diagnose its existence is not likely to cause so much general febrile disturbance as is noted at the end of the first week of an attack of typhoid fever. This maximum of fever and minimum of local disturbance, though associated clinically, are not likely to be associated causally. It cannot, indeed, be said that so important a lesion causes no general disturbance and has no share in the production of the fever, but it is evident that its presence is not sufficient to explain and account for the marked rise of temperature noted during the first week of the disease. There must be some other agency at work: but the sole agency with which we have to do is the bacillus. There must, therefore, be some action of the bacillus other than its local action on the intestinal glands, in virtue of which it causes the general febrile disturbance.

In dealing with the action of the bacillus on the intestinal glands we are dealing solely with the action of the parasite on its nidus. But there is also the general action of the organism on the system; and that is what we have to do with in considering the pathogenesis of the general febrile disturbance. The first bacillus which enters the system from without is entirely inert until it is vivified and roused into activity by contact with its nidus in the intestinal glands. Here it is rapidly reproduced and in a short time, as gland after gland is invaded, hundreds. thousands, and ultimately millions of the specific organisms of typhoid fever pass from the nidus into the general circulation through the numerous lacteals and veins with which the affected glands are so abundantly supplied, in a network of which indeed they lie embedded. But be it specially noted that the mass of the organisms which thus pass into the circulation are not mature bacilli but young germs which have just been reproduced, have just entered on their separate existence, and whose organic growth has yet to be completed. Not only in the affected glands but all over the body, in the minute structure of all its tissues, this organic growth goes on and the poison passes from the germ state to that of the mature bacillus. It is the action resulting from the organic growth in the tissues of these millions of organisms that gives rise to the general febrile disturbance associated with the development of the bowel lesion.

The action of the bacillus is thus a dual one : (1) there is the specific local action of the parasite on the intestinal glands; and (2) the general action of the organism on the blood and tissues. The bowel lesion does not cause the fever and the fever does not cause the bowel lesion. They are conjoint results of this double action of the bacillus.

To form a just estimate of the tremendous morbific power of these organisms and of their capacity to produce disturbance we must have some idea of the enormous extent to which they are reproduced and the immensity of the numbers with whose action we have to deal, for it is the vastness of their number rather than the potency of the individual that serves to explain the phenomena to which they give rise. Suppose that each bacillus produced ten others and that one day was the time requisite for the process of reproduction we should from one bacillus have in ten days 1000,000,000:thus, on the first day one; on the second day 10; on the third day 100; on the fourth day 1000; on the fifth day 10000 ; on the sixth day 100,000 ; on the seventh day $1,000,000$; on the eighth day $10,000,000$; on the ninth day $100,000,000$; and on the tenth day $1,000,000,000$. Let us take a more modest estimate and suppose that each bacillus produced only four others in 24 hours, we should have the following result: on the first day one; on the second day four; on the third day 16; on the fourth day 64 ; on the fifth day 256 ; on the sixth day 1024 ; on the seventh day 4096 ; on the eighth day 16,384 ; on the ninth day 65,536 . on the tenth day 262,144 ; on the eleventh day $1,048,576$; on the twelfth day $4,194,304$; on the thirteen th day $16,777,216$; and on the fourteenth day $67,108,864$. Wven at that rate we should have in a fortnight from one bacillus more than $67,000,000$. Suppose 12 instead of 24 hours to be the time requisite for the reproduction of the germs their rate of increase would be doubled. The mind can hardly grasp the number of bacilli which may thus be launched into the circulation.

The action which culminates in this result and in the symptoms of typhoid fever begins at the beginning of the period of incubation, the time at which the poison is re. ceived into the system. At first from the paucity of the numbers the rate of increase is comparatively slow and the number of bacilli brought into action is not sufficient to cause disturbance of the system. That is what is taking place during the period of incubation-that is, indeed, what constitutes that period. But it is evident that a time must come when the quantity of poison in the system is so great that it cannot be reproduced four-fold without causing almost an explosion of germs in the system. The onset of this inevitable event terminates the period of incubation and ushers in the stage of invasion. A glance at the figures given will show how certeinly this must occur. The reproduction, slow at first, speedily becomes very rapid. Taking the first set of figures the number of bacilli produced at the end of the first five days is 10,100 , by the end of the second five it is 1,000,000,000 Taking the second set, at the end of the first week the number is under 5000 ; by the end of the second week it is over 67000,000 .

It is the organic growth in the tissues of these vast hordes of bacilli that causes the $f \in v e r$ and general disturbance noted in typhoid fever. How do they do this? Rise of temperature may be produced in one of two ways: (1) by increased activity of the process by which heat is formed; or (2) by diminished activity of the process by which its formation is inhibited and kept within due bounds. That due to impaired inhibition is illustrated hy hyperpyrexia and the increased body heat which results from some lesions of the brain and cervical cord. That due to increased production is exemplified by that with which we are now dealing. ${ }^{2}$ But rise of temperature is not the only symptom of such fever. Wasting of the nitrogenous tissues, increased elimination of urea, increased rapidity of the circulation, thirst, dry skin, and scanty urine,- - these with rise of temperature are the essential phenomena of fever.

The essential and distinctive feature of an organism is its action on its environment. The action of an organism such as the typhoid bacillus on its environment in the tissues consists in the consumption of nitrogen and water-a consumption which, in virtue of their vast numbers, must be very great. Now when we come to examine minutely into the various phenomena of the febrile state as it occurs in the specific fevers we find that they ultimately resolve themselves into two-increased consumption of nitrogen and increased consumption of water. From these two all the others result. Rise of temperature, quickening of the circulation, wasting of the nitrogenous tissues, increased elimination of urea, thirst, loss of appetite, dry skin, scanty urine all owe their existence to the consumption by the contagium of the nitrogen and water destined for the tissues.

2 See "Fever, a Clinical Study," by T. J. Maclagan, M.D. (J. \& A Churchill, 1888). 
For their consumption by these hosts of bacilli represents a direct loss to the tissues. Occurring in the nitrogenous tissues it gives rise to the rapid wasting of these tissues noted during an attack of typhoid fever. Occurring in the brain it causes the headache of the earlier and the wandering of the later stages. Other and necessary results of this consumption by an external agency of the water destined for the tissues are those indicative of a short supply-thirst, dry skin, and scanty urine. The supply may be ample, but it is tapped en route by the bacilli and does not reach the tissues in sufficient quantity.

The seat of normal tissue metabolism is the minute structure of the tissues. That, too, is the seat of the abnormal action which we are now considering. Tissue metabolism consists (1) of the taking up of new material from the store albumin, to form fixed or organ albumin ; and (2) of the giving off from the tissues of their worn-out organ albumin, which passes back into the circulation and is ultimately eliminated as urea. These actions are simultaneous, neither preceding the other. The bacilli lay hold of the nitrogen of the store albumin at the moment when it is about to be appropriated by, and incorporated in, the tissues as organ albumin. The simultaneous action by which the worn-out organ albumin passes back into the circulation is equally far advanced. Nothing intervenes to prevent this, it goes on only more actively in consequence of the increase of metabolism, and thus is brought about the physiological anomaly that lessened consumption of nitrogen by the tissues is accompanied by increased elimination of nitrogen by the kidneys.

The matter may be briefly put thus: What is the cause of the wasting of the nitrogenous tissues? The consumption by millions of bacilli of the nitrogen destined for their nutrition. What is the cause of the thirst, dry skin, and scanty urine? The consumption by the same bacilli of the water destined for the tissues. What is the cause of the rise of temperature and quickening of the circulation? The increased metabolism which necessarily results from this action going on in the minute structare of the nitrogenous tissues, the chief seat of heat formation. Thus all the essential phenomena of the febrile state as it presents itself in typhoid ferer are traceable back to these two agenciesincreased consumption of nitrogen and increased consumption of water ; and these in their turn result from the action necessarily attendant on the organic growth of vast numbers of bacilli in the structure of the tissues.

Other symptoms noted during the course of the fever are equally explained on this view of the mode of action of the bacillus. The headache of the earlier and the delirium of the later stages of the fever are due to cerebral inanition consequent on the consumption in the brain tissue of the nitrogen and water destined for the nutrition of that organ. The softening of the cardiac muscle and the changes noted in it after death are due to the same cause acting in its structure.

In typhoid fever, as in the other specific fevers, the soda salts are retained in the system or are eliminated in much diminished quantity, while the potass salts are eliminated in increased quantity. The soda salts exist chiefly in the liquor sanguinis; they are retained because the bacilli in taking up the nitrogen and water of the liquor sanguinis take up also the soda salts in it. The potass salts exist chiefly in the tissues; they are eliminated in increased quantity because there is increased tissue disintegration. All these actions go on and all the febrile symptoms continue so long as the bacillus is being reproduced and come to an end only with the cessation of that process. But why does it cease? Why do these organisms not go on growing as long as any nitrogen and water remain for them to consume? And why does anyone ever recover? The fever thus caused comes to an end when the bacilli cease to be reproduced, and their reproduction ceases when the nidus is exhausted, that is, when all the glands which form the nidus have been destroyed. The course of events shows that on an average it takes five weeks more or less for the completion of this process, two weeks occupied by the period of incubation, and three by the febrile stage. Thus all the essential phenomena of typhoid fever, its rise, course, and decline, are causally associated with the life-history of its bacillus in the system.

Recrudescence of the fever.-It has already been pointed out that the intestinal glands are not all affected at the same time and that some reach the stage of sloughing and ulceration, while others more tardily affected are still at the stage of hardening and thickening. It not unfrequently bappens that some glands do not become involved in the morbid process till so late in the disease that the fever caused by those already affected is on the decline or may even have come to an end and the temperature be normal before the fresh lesions are sufficiently advanced to add their quotum to the general disturbance. Their appearance on the scene is heralded by a renewed rise of temperature and a recrudescence of the fever. Sach recrudescence may occur towards the end of the third week or at any subsequent period. It means fresh glandular lesions, the launching into the circulation of fresh shoals of bacilli and consequent recrudescence of the fever, with a renewal of all the symptoms local and general.

Cases are not uncommon in which, by a repetition of these recrudescences, the fever is prolonged into the fifth or sixth week, it may be without any material addition to the gravity of the symptoms other than that which comes from the prolongation of a disease which is so exhausting and so full of hazards. The latter part of these cases is really made up of a succession of shori, attacks following each other so quickly that the one almost dovetails into the other. Each is indicative either of the invasion by the bacilli of one or more fresb glands or of a fresh absorption of septic material.

Relapses.-Sometimes the invasion of the latest affected glands is so retarded that instead of an apyretic interval of only 12 or 24 hours we have six or eight, or even 10 or 12 , days of apyrexia and apparent convalescence. The fever is then said to relapse. The pathogenesis of the relapse is the same as that of the recrudescence; the difference is the length of the period of apyrexia which separates the second from the first attack. This period of apyrexia is really the period of incubation of the second attack. But the beginning of that period is contemporaneous with the later days of the primary seizure, so that the poison which gives rise to the relapse has really begun its action before the first attack is at an end, and the two, though clinically distinct and separate, are pathologically one.

It has been supposed by some that relapses may be due to errors of diet, to getting up too soon, or to other indiscretion. But the relapse is as truly an attack of typhoid fever as was the primary seizure. Nothing could cause this except the typhoid bacillus. Moreover, the poison which causes it has really begun its action before the first attack is at an end and while the patient was still confined to bed. The relapse is of shorter duration and generally milder than its predecessor. That is what, on this view of its pathogenesis, we might expect, for the field of operation of the bacilli is much curtailed by the destruction during the first attack of so many of the intestinal glands. There is a more scanty nidus, fewer glands to suffer, and a consequent reduction both in the severity of the local lesion and the duration of the febrile symptoms ; these seldom last more than a fortnight, often less. The shorter duration is due to the same cause as the lessened severity ; there are not glands enough to cause three weeks of fever. But though the relapse is generally milder than the first attack the rule has a good many exceptions, for every now and then a relaose proves fatal. In connexion with this subject it has to be borne in mind (1) that the relapse comes when the sufferer is already weakened hy the first seizure; and (2) that one lesion in the bowel suffices for the production of hæmorrhage and perforation and of a fatal issue.

It is mainly in consequence of their shorter duration that relapses are less fatal than first attacks, for the dangers of typhoid fever necessarily increase as the disease goes on. This shorter duration is due to the fact already indicated, that the nidus of the parasite, the field of operation of the bacillus, is materially reduced in extent by the destruction of the glands involved in the first attack.

It is a demonstrated fact that during the relapse glands suffer which bad escaped oll the risks to which they were exposed during the primary seizure. It occasionally happens that some even run the gauntlet of a second attack without suffering, for second and even third relapses, each with fresh lesions, are not unknown. How come they to escape? How is it possible for any glands to escape invasion by some of the vast number of bacilli reproduced during the course of the fever? Were this poison liquid and equally diffused through the blood it would be impossible for any glands to escape ; but it is not liquid and diffusible, but particulate and indiffusible, and therefore not of necessity equally distributed through the blood. The germs reproduced in the intestinal glands may pass into the 
general circulation either through the lacteals or through the superior mesenteric vein. But the bacilli in the blood can gain access to the glands only by way of some of the branches of the left or convex side of the superior mesenteric artery. The chance that no bacilli may ever reach some one particular branch of this artery which supplies a given inch or two of the bowel and a given half-dozen glands is quite an appreciable one. The clinical history of typhoid fever and the occurrence of relapses with fresh lesions shows that this chance occasionally becomes a reality.

Such is the explanation of the mode of action of the typhoid bacillus. The local disturbance in the intestinal glands is the result of the reproduction of the parasite in its nidus. The general febrile disturbance is the consequence of the organic growth in the tissues of the millions of organisms thus reproduced in these glands. Neither action would in itself be disastrous or be followed by serious consequences but for the fact that the trouble in the intestinal glands ends in sloughing and suppuration. These two processes bring into play other morbific agencies which are much more potent for evil than the typhoid bacillus. Before the glands slough there are no serious bowel symptoms and nothing to call special attention to the bowel. As already pointed out, the serious bowel symptoms which are apt to show themselves in the later stages of the ferer are caused not by the typhoid bacillus but by the varions organisms which come into play with the processes of sloughing and suppuration. ${ }^{3}$ To the absorption of these organisms into the system are to be ascribed the prolonged duration of the fever in many of those severe cases which run on into the fourth and fifth weeks, the high range of temperature noted in their later stages, and all the alarming symptoms by which it is accompanied. The high fever, the feeble rapid pulse, the dry tongue, the muttering delirium, and general condition of typhoid prostration which characterise the later stages of severe cases are the symptoms not so much of typhoid fever as of septic poisoning. They occur in the advanced stages of bad cases of typhoid fever, but they are due not so much to the direct action of the typhoid bacillus as to that of the various forms of cocci associated with the sloughing process in the bowel. It is the absorption of these into the system and their reproduction there that causes the alarming symptoms of severe cases and makes typhoid fever so serions and so fatal a disease. There are degrees of this as there are degrees of typhoid fever. The sloughs may be comparatively few and come away without giving trouble. The more severe the bowel lesion the more extensive is the sloughing, the deeper the ulcers penetrate into the gut, and the more likely we are to have the poison absorbed into the system.

Gangrenous ulceration in any part of the body is apt to cause septic poisoning; that is, indeed, the chief danger of such ulceration. The risk of this is specially great in typhoid fever, first because the number of ulcers and consequent foci of absorption are so numerous, and second because the affected glands are situate in a network of veins and lacteals whose function it is to take up and carry into the system the materials presented to them.

Though all the symptoms of typhoid fever are ultimately to be traced back to an action set agoing by the typhoid bacillus, not one of its serious symptoms or special dangers is due to the direct action of that bacillus. The dangers attendant on the bowel lesion, diarrhœe, hæmorrhage, and perforation result from the destructive action on the mucous, muscular, and peritoneal coats of the organisms associated with the processes of sloughing and suppuration. The alarming general symptoms which are noted in the later stages of severe cases -high temperature, feeble cardiac action, delirium, and prostration-are due not so much to the growth in the tissues of the typhoid bacillus as to the superadded growth of the millions of cocci which are absorbed into the system from the sloughing glands by the veins and lacteals among which these glands are embedded.

Cadogan-place, S.W.

3 The Laxcht, Dec. 8th, 1900, p. 1639.

ForetgN UNIVERITY INTELLIGENCE.Brussels: Dr. Bayet has been appointed Professor of Clinical Dermatology, and Dr. Cheval Professor of Olinical Laryngology.- Halle: Dr. Fritz Haasler, privat-docent of surgery, has been granted the title of Professor.

\section{d) âturrox}

or

\section{H OSPITAL PRACTICE, BRITISH ANI FOREIGN.}

Falla autem est alia pro certo noscendi via, nist quamplurimas ot morborum ot dissectionnm historias, tum aliorum tum propria. sollectas hubere, et inter
corb., lib. iv. Procemium.

\section{ST, GEORGE'S HOSPITAL.}

\section{A CENTRAL TUMOUR OF THE SHAET OF THE RADIUS TBEATED BT ENUCLEATION.}

(Under the care of Mr. MarmadukE Sheird.)

THE advisability of the employment of enucleation for the treatment of a tumour of bone must depend ohiefly on the nature of the growth. For non-malignant tumours this operation may safely be performed, and it is applicable even to cases of central chondromata, in which formerly amputation was always considered necessary. But in any form of malignant growth there is great risk of recurrence. And although in some cases of myeloid sarcoma no recurrence has been recorded after enucleation this must sometimes be due to the case having been reported soon after the operation. Still a sufficient number of cases have been put on record in which the less malignant forms of malignant growths occurring in the interior of bones have been treated by enucleation, and no recurrence has occurred even after several years. The treatment is therefore advisable whenever the slowness of growth or the microscopic structure of the tumour suggests that the malignancy is slight. For the notes of the case we are indebted to Mr. C. $R$. Keyser, F.R.C.S. Eng., surgical registrar.

A small, spare, and excessively anæmic woman, aged 19 years, was admitted into St. George's Hospital on Sept. 18th, 1900, under the care of Mr. Marmaduke Sheild, suffering from a tumour of the right forearm. The history was as follows. Ten years ago, whilst swinging on a ladder, she twisted her right arm and "broke a small bone." On the removal of the splints some weeks later the swelling which she on admission complained of was discovered. It had gradually increased in size and gave rise to pain at times in the arm and fingers. She was positive that the tumour was of 10 years' duration. On examining the right forearm there was an oval-shaped tumour, obviously an enlargement of the radius, about midway between the elbow and the wrist, but projecting mostly towards the radial side of the arm. It was of stony hardness, with some increase of heat on the surface, and there were many dilated veins coursing over it. It was not adherent to the skin, and no softening, bruit, pulsation, or egg-shell crackling could be detected. In length it measured three inches and the circumference at its widest part was seven and a half inches. The muscles of the forearm and hand were somewhat wasted, but she stated that the right hand was just as useful and as strong as the left. Aching pain was constantly present. It was thought at "consultations" that the tumour was either a very slowly growing (so-called) myeloid sarcoma, an enchondroma, an old fracture with excessive formation of bone, or some rare form of osseous cyst, such as hydatid or sanguineous cyst, but in any case that it ought to be explored and dealt with according to what was found.

On Oct. 4th. ether having been given, Mr. Sheild applied an Esmarch's bandage to the limb and then made an incision about five inches long on the radial side of the tumour on its dorsal aspect and exposed the bone and trephined it. The wall of the tumour was very thin and its cavity was partially divided up into loculi by thin bony septa. These can be well seen in the striking skiagram which was taken at the hospital by Mr. Addyman in charge of the Roentgen-ray apparatus. The bony wall of the tumour was cut away for about four inches and the contents, which were soft, and yellowish in colour, were scooped out with a sharp spoon. The small lower growth seen in the skiagram was left alone. The inner aspect of the hollow was then thoroughly cauterised and swabbed over with pure 